\title{
Fusing of optimized intelligence models by virtue of committee machine for estimation of the residual shear strength of clay
}

\author{
Mahsa Gholami $^{1} \cdot$ Asadollah Bodaghi $^{2}$
}

Received: 24 February 2016/ Accepted: 27 February 2016/Published online: 10 March 2016

(C) Springer International Publishing Switzerland 2016

\begin{abstract}
Owing to high dependency of landslide stability to residual shear strength (RSS) of clay, provide a sophisticated strategy for modeling of this parameter is advantageous. This paper present strategy based upon fusing of optimized intelligence models for estimation of RSS of clay as a function of readily available data. The developed model is achieved through implementing two following steps. In the first step, two optimized models including optimized neural network, and optimized fuzzy logic are developed for estimation of RSS of clay. Optimizing method which implanted in predictive models for improving those performance is bat-inspired algorithm. In second step, committee machine $(\mathrm{CM})$ is employed for combining outputs of aforementioned optimized models. Bat-inspired is incorporated in CM for determining optimal contribution of optimized elements in final prediction. The superior performance of $\mathrm{CM}$ rather than its elements is ascertain through those evaluation based on statistical criteria. Results of this study infer that proposed methodology provide an alternative way for making quantitative formulation between RSS of clay and its index properties.
\end{abstract}

Keywords Residual shear strength (RSS) - Optimized neural network (ONN) · Optimized fuzzy logic (OFL) · Committee machine $(\mathrm{CM}) \cdot$ Bat-inspired algorithm (BA)

Mahsa Gholami

mahsagholami72@yahoo.com

1 Department of Civil Engineering, Factually of Engineering, Bu-Ali Sina University, Hamedan, Iran

2 Hamedan Regional Water Company, Hamedan, Iran

\section{Introduction}

Landslides are prevalent natural hazards taking place in most parts of the world and have negative economic impacts (Clague and Stead 2012; Skempton 1964). Residual shear strength (RSS) of clay has been thought as one of critical parameter in evaluating the stability of landslide. This importance is highlighted in sensitive clays wherein variation of shear strength from peak to residual states is considerable. At a residual state, the great strain is taken to clay thereby crashing its previous bond and annihilating prior fabric as well. Moreover at the residual strength, clay has negligible cohesion and extremely tend to remoulding (Mesri and Shahien 2003; Colotta et al. 1989; Wesley 2003; Tiwari and Marui 2005; Kaya and Kwong 2007). Obtaining this parameters in laboratory is required the plenty of time and effort. Therefore, reliable prediction of RSS of clay is very helpful for evaluation of landslide stability. The great complexity and difficulties encountered in prediction of RSS of clay have motivated researchers to apply intelligent models for solving this problems. To date, a variety of intelligence based models are available in the literature for exploring complex nonlinear underlying relationships between the RSS of clay and clay index (Das and Basudhar 2008; Khan et al. 2015; Das et al. 2011). The basic idea of these models is to find out the relation between input/output through mimicking the neural behavior of brain. These models, according to the input parameters, can be classified into two groups: model which employed clay friction (CF), and differential plasticity index $(\Delta \mathrm{PI})$ as inputs and model that consider liquid limit (LL), plasticity index (PI), CF, and $\Delta \mathrm{PI}$ as a model input. In first group, Das and Basudhar (2008) employed artificial neural network to correlate RSS of clay to $\mathrm{CF}$, and $\Delta \mathrm{PI}$. They concluded that ANN can obtain adequate results in 
prediction of RSS of clay. In second group, two papers published recently for demonstrate the capability of intelligence models for estimation of RSS of clay (Khan et al. 2015; Das et al. 2011). These papers argue the capability of intelligence based model for identifying the functional dependency between RSS of clay and aforementioned four clay index. Although these models have an acceptable accuracy, present a novel for estimation of RSS of clay is valuable. This study aim to introduce smart model for prediction of RSS of clay using of following variable as model inputs: LL, PI, CF, and $\triangle \mathrm{PI}$. A flowchart of employed strategy in this study is illustrated in Fig. 1. As it can be seen, estimation of RSS of clay from input parameters is firstly done via intelligence models optimized with bat-inspired algorithm (BA) [optimized neural network (ONN), and optimized fuzzy logic (OFL)]. Subsequently, the outputs of optimized models are integrated through concept of committee machine (CM). Each optimized models is associated with a weighting factor to determine the final prediction of RSS of clay. Optimal values of these weight factors are calculated by virtue of BA. Results of this study prove that, compared with individual intelligence models, $\mathrm{CM}$ has a superior performance. Owing to simplicity and efficiency of CM model, it can be used as a good tool for estimating of RSS of clay.

\section{Theory: committee machine}

Referring to Fig. 1, in the first stage of current study, two optimized models including ONN, and OFL are employed as a committee members to correlate the input parameters into RSS of clay. Then CM is yielded through integrating the results of aforementioned experts with BA. This combination is worth due to that model is generated which reap the benefits of two intelligence models and improve those generalization performance. CM has a parallel structure which is capable of assembling the outputs of various experts to obtain an overall decision with improved accuracy (Ansari 2014; Gholami et al. 2014a, 2015b; Asoodeh
Fig. 1 The flowchart of the proposed scheme for obtaining residual shear strength of clay. As seen in this figure, in first stage two BA optimized intelligence are employed for estimation residual shear strength of clay. Then, committee machine with BA algorithm is used for combining of results of aforementioned models

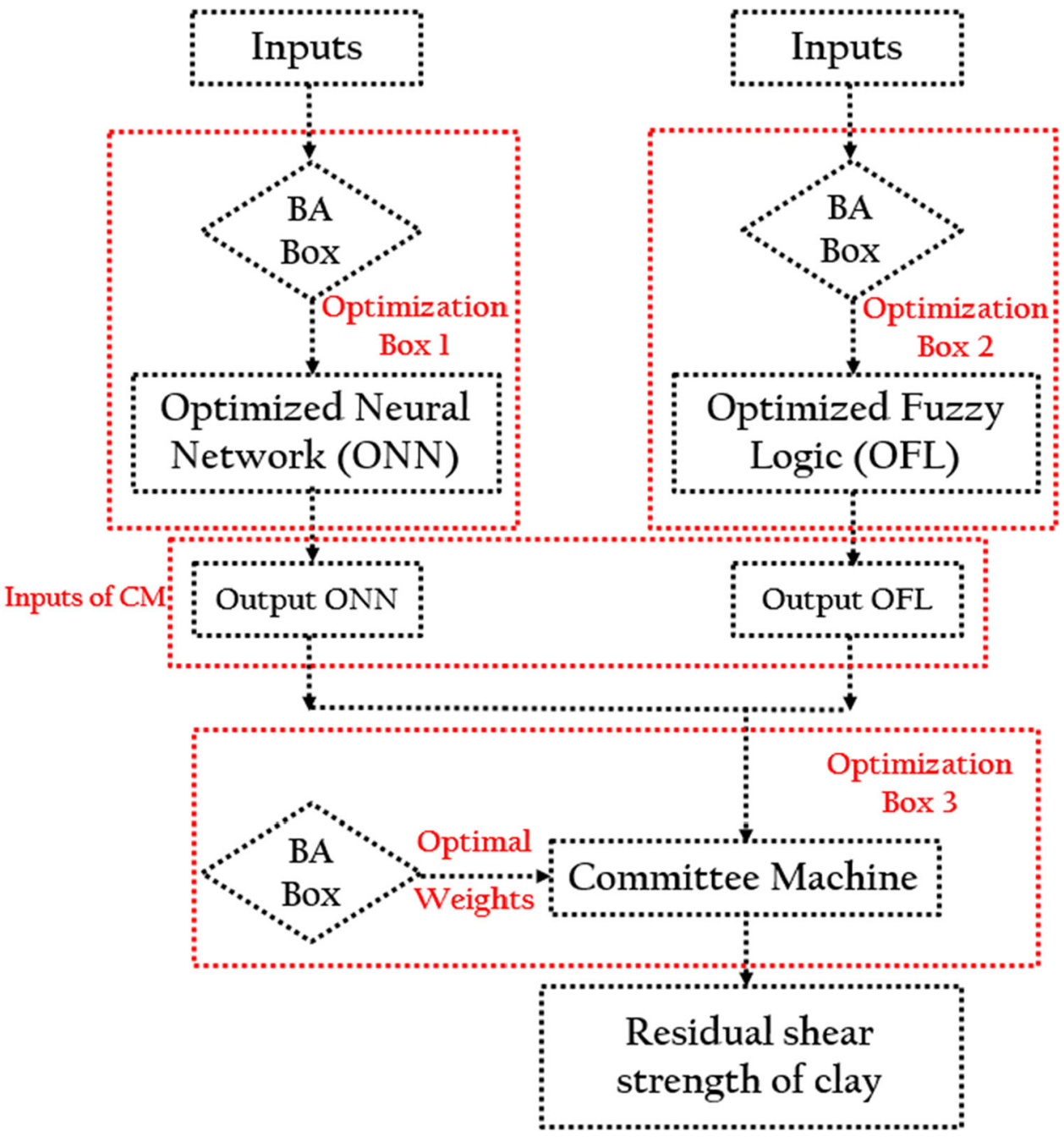


and Bagheripour 2012a, b; Asoodeh et al. 2014a; Kadkhodaie-Ilkhchi et al. 2009a, b). The details of optimized models for constructing CM are discussed in the following sections.

\section{Optimized neural network}

The artificial neural network intelligence based model simulated the working of the human brain and nervous system for learning the relationship between dependent and independent variables (Baughman and Liu 1985). The architecture of the NN consists of one input, one hidden and one output layers. Input layer gathers the input data. Output layer produces the output data. Hidden layer extracts the underlying dependency between input/output data. Number of neuron in input and output layers is equal to number of input and outputs parameters, respectively. Optimum number of neuron in hidden layer for achieving model which give the best results is determined via try and error approach. In recent years, this approach emerges as a new predictive scheme that achieved considerable research interest owing to its simplicity and efficiency (Mäkisara et al. 2005). However, when applied to regression problems, NN suffers from trap into local optima and cause the premature convergence phenomenon, this may lead to great prediction error from the constructed model on testing data. To remedy this fault, different researchers try to embed optimization algorithm in NN formulation for optimize its parameters and achieve acceptable improvement (Zargar et al. 2015a; Gholami et al. 2014b, 2015a; Asoodeh et al. 2014b). In current study, BA algorithm is adopted for optimization of neural network parameters.

\section{Optimized fuzzy logic}

Fuzzy logic is mathematical based model developed by Zadeh (1965) for handling uncertainty. This method aim at using "degrees of truth" in place of the usual "true or false" logic calculation. Each fuzzy set is represented by membership function. This methodology applied to solving of several nonlinear problems in engineering task (Gholami et al. 2014c). In an FL, there are three main parts including fuzzifier, inference engine (or fuzzy rule base) and defuzzifier. The purpose of fuzzification is to map the input data to values ranging from 0 to 1 using a set of input MF. The main part of the FL is inference engine which consists of a fuzzy rule. The outputs of all of the fuzzy rules are combined to obtain one fuzzy output distribution and transfer into a crisp output using output MF in defuzzifier process. There are two main type FL system, Mamdani and Sugeno. In this study Sugeno is employed. This mathematical approach attempt to finding underlying relation between dependent and independent parameters. Achieving this will require determine the optimal values of FL parameters which can be attained through including of optimization model in those formulation. Various efforts have been made to coupling the optimization algorithm and fuzzy model (Zargar et al. 2015b; Asoodeh et al. 2015; Afshar et al. 2014). These studies demonstrate the high efficiency of optimization algorithm in enhancing the performance of fuzzy logic model. In the research documented here, BA is included in fuzzy logic formulation for finding out optimal values of its parameters.

\section{Bat-inspired algorithm}

The BA is a mathematical simulation of echolocation behavior of the bats (Yang 2010). All bats fly randomly and use echolocation to find position of a prey or food. In the BA, each position of bats is considered as a probable solution and the goal is find the position of a prey (best solution). To search for a prey, bats fly randomly with velocity $v_{i}$ and fixed frequency $f$ varying wavelength $\lambda$ and loudness $A_{0}$. Each bat is randomly allocated a frequency that is drawn with a uniform distribution in range of $\left[f_{\min }\right.$, $\left.f_{\text {max }}\right]$. The emitted pulses of bats have a rate of pulse emission $r_{i}$ in the range of $[0,1]$, dependent on the proximity of foods. Each bat takes a random walk creating a new position based on the best selected current position of a prey. Therefore the positions, frequency and velocity of the bats are updated in each algorithm iteration. Updating process in order to find best solution is continues until a certain stop condition is met (Ansari and Gholami 2015). In this study, the stop condition is maximum number of iteration. In this study, the stop condition is maximum number of iteration. In this research, BA is employed for achieving three important task: (1) determining the optimal value of neural network parameters including weight and bias, (2) determining the optimal value of fuzzy logic parameters, (3) computing the optimal contribution of committee elements in final prediction.

\section{Data input/output space}

To build predictive model for estimating the RSS of clay, dataset containing the reliable experimental data is collected from open source literature. Referring to Das et al. (2011), paper, RSS of clay can be determined as a function of parameters viz. LL, PI, CF, and $\triangle \mathrm{PI}$. Hence, aforementioned parameters are used as input variables for estimation of RSS of clay. Dataset is partitioned randomly into two distinguished parts: a training set with $75 \% \quad(98$ sample points) of the data and a testing set with the $25 \%$ (33 sample points) of the data. Training set is employed for constructed model, while testing set is utilized for 
Table 1 Statistical description of experimental data applied in current study for training and testing model

\begin{tabular}{lllrll}
\hline Parameter & LL & PI & \multicolumn{1}{c}{$\Delta$ PI } & CF & RSS \\
\hline Minimum & 22 & 4.5 & -94.89 & 0.4 & 5.5 \\
Maximum & 213 & 132 & 29.07 & 91 & 39 \\
Average & 68.79 & 34.21 & -1.41 & 30.98 & 16.47 \\
SD & 28.67 & 19.8 & 13.36 & 19.37 & 8.02 \\
\hline
\end{tabular}

Table 2 Regulations done before run of bat-inspired algorithm for optimizing all proposed models including: ONN, OFL and CM

\begin{tabular}{llll}
\hline Parameter & \multicolumn{2}{l}{ Value } & \\
\cline { 2 - 4 } & ONN & OFL & CM \\
\hline Population size & 100 & 100 & 20 \\
Maximum iteration & 1,000 & 1,000 & 1,000 \\
$A_{0}$ & 0.5 & 0.5 & 0.5 \\
$r_{0}$ & 0.5 & 0.5 & 0.5 \\
$f_{\min }$ & 0 & 0 & 0 \\
$f_{\max }$ & 2 & 2 & 2 \\
\hline
\end{tabular}

evaluated the robustness and reliability of constructed model. The statistical description of utilized dataset is given in Table 1.

\section{Results and discussion}

An accurate determination of the RSS of clay is significant to execute a realistic evaluation of landslide stability. In first stage of this study, two optimized model including ONN, and OFL are used for modeling of RSS of clay. The integrating of two optimized models by virtue of CM is then employed for estimation of RSS of clay. The performance and accuracy of constructed model are figured out using following indexes as statistical criteria: correlation coefficient $(R)$, and root mean square error (RMSE). Generally, predictive model with lowest value of RMSE and highest value of $R$ giving superlative performance compared to its competitors.

\section{Optimized neural network}

In this part of current research, ONN is carried out for construct predictive model to estimate the RSS of clay. As mentioned in previous section (Sect. 2.1), for obtaining neural network with highest performance, optimal values of weights and biases should be determined. Therefore, to achieve aforementioned objective, BA is implanted into neural network formulation. Regulated parameters of the BA for run the ONN are presented in Table 2. Moreover, identification of appropriate network configuration has a
Fig. 2 Influences of the number of neuron in hidden layer in accuracy of optimized neural network for a training data and b testing data. This figure demonstrated that optimum number of neuron for achieving best optimized neural network model is 4

\section{Training data}

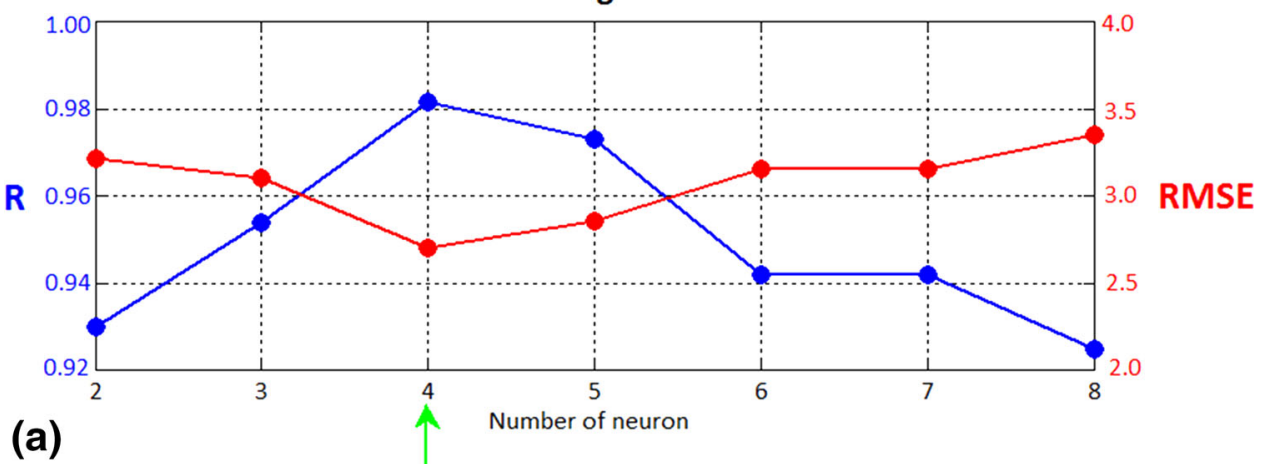

Testing data

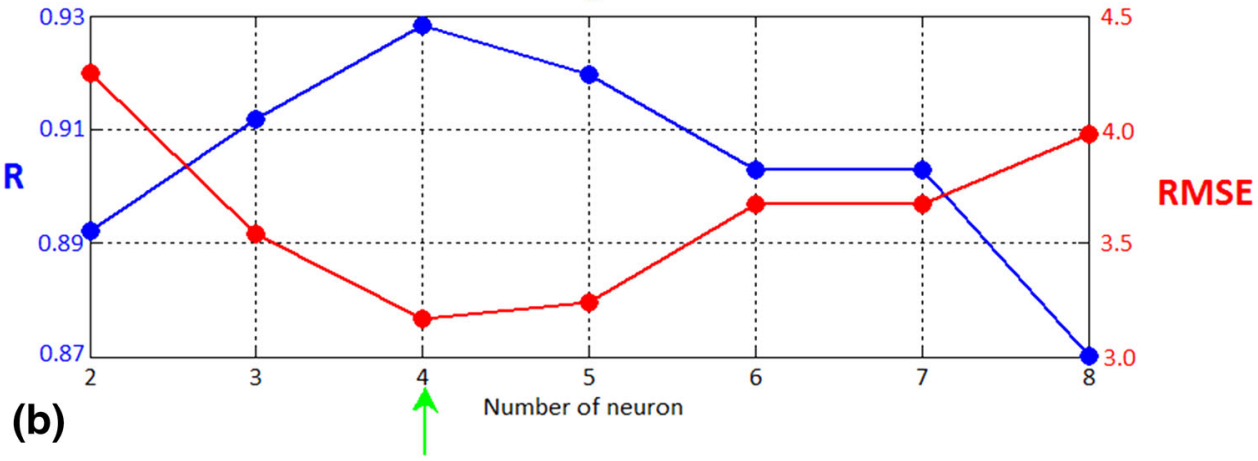


Fig. 3 Plot of mean and best fitness (MSE) of BA versus generation for a optimizing neural network, b optimizing fuzzy logic, and $\mathbf{c}$ determining the optimal contribution of optimized intelligence in final prediction
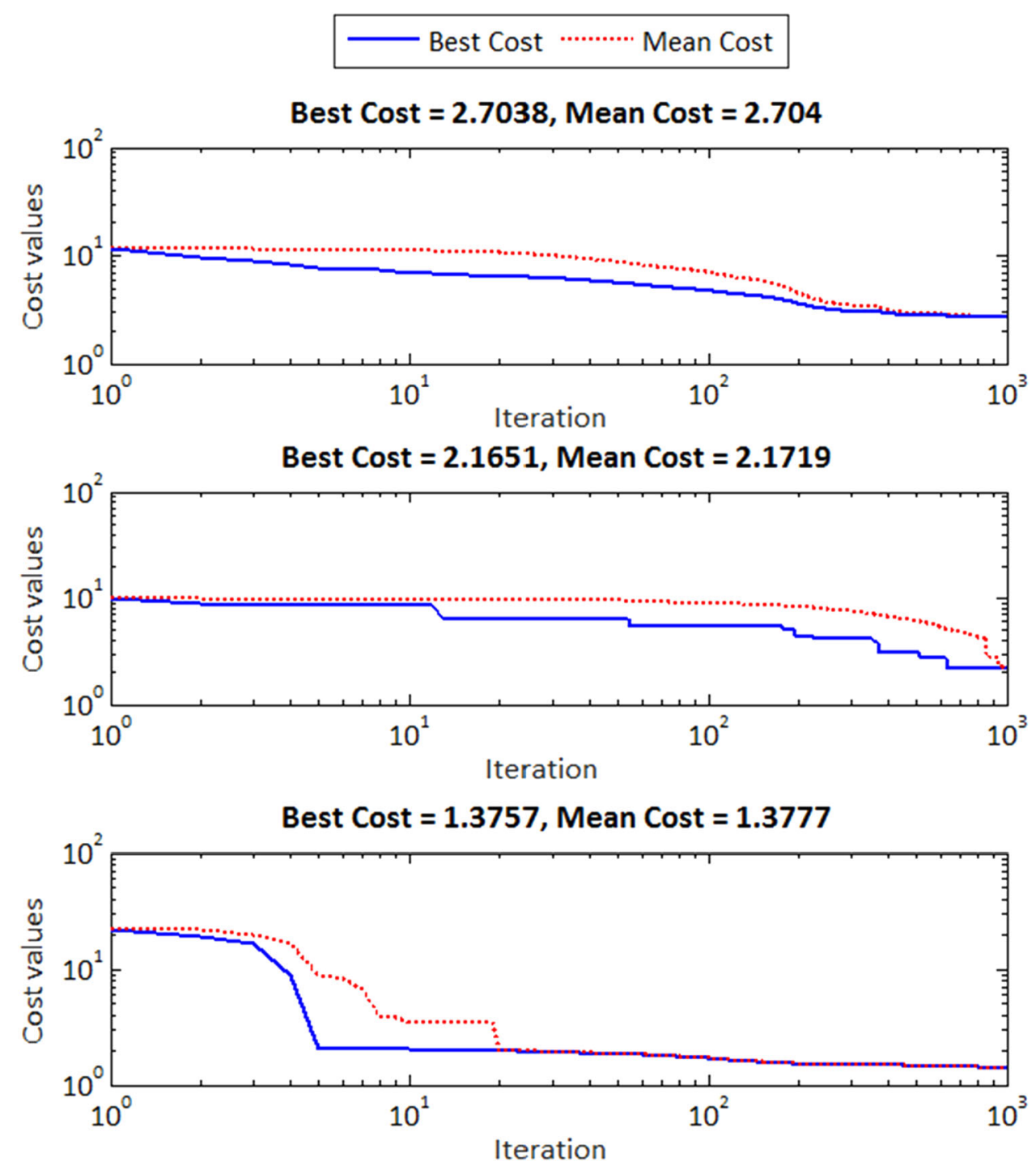

Table 3 The optimal values of weight and bias which determined through embedding BA in neural network formulation

\begin{tabular}{lrrrrr}
\hline Layer & \multicolumn{2}{l}{ Weights } & & Biases \\
\cline { 2 - 5 } & Input 1 & Input 2 & Input 3 & Input4 & \\
\hline Hidden layer & & & & \\
Node 1 & 2.0952 & 2.1847 & -4.5306 & 6.1883 & -5.1861 \\
Node 2 & 0.0327 & 4.5530 & 0.1622 & 3.2595 & -1.4966 \\
Node 3 & 8.0616 & 4.3764 & -1.2756 & 3.6783 & -3.8013 \\
Node 4 & -1.6399 & -5.9073 & -0.0253 & -1.4043 & -5.2054 \\
\hline Layer & Weights & & & & Biases \\
\cline { 2 - 5 } & Node 1 & Node 2 & Node 3 & Node 4 & \\
\hline Output layer & & & & \\
Node 1 & 1.8234 & -1.5673 & 0.1341 & 0.4706 & 0.0001 \\
\hline
\end{tabular}

crucial role on capability of neural network in for handling modeling task. Hence, the appropriate number of neuron in hidden layer of should be selected. To gain this purpose, methodology based on try and error approach is applied and those results are depicted in Fig. 2. As can be seen in this figure, optimum number of neuron for reaching better result in training and testing phase is 5. Figure 3 a shows run of BA for optimizing $\mathrm{NN}$ during 1,000 iterations. The optimal values of weight and bias is given in Table 3 . The estimated outputs from the ONN model are compared to the real values of RSS of clay in term of scatter plot in Fig. 4a. This figure show the satisfactory correlation between estimated results of ONN and real value of RSS of clay. Figure 5 illustrated that difference between measured and estimated RSS of clay for each testing sample point. High correlation between estimated results of ONN and real value validate good precision and accuracy of the model. 

estimated residual shear strength of clay for the a optimized neural network model, b optimized fuzzy logic model, and c committee machine model. This figure demonstrated that committee machine has a better results rather than its elements
Fig. 4 Observed versus
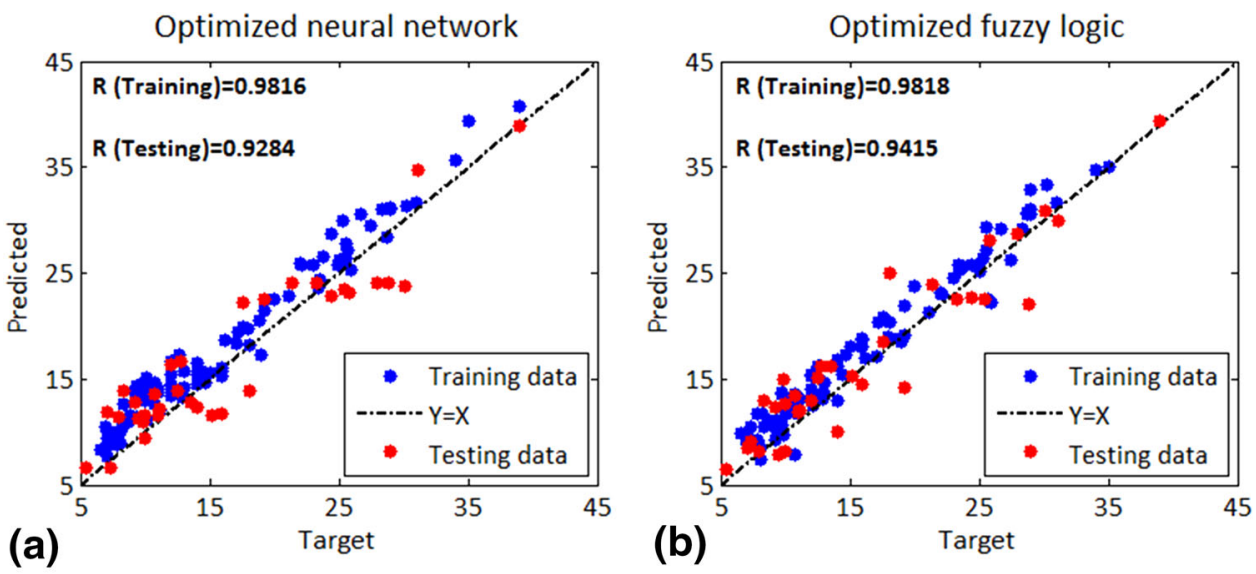

Committee machine

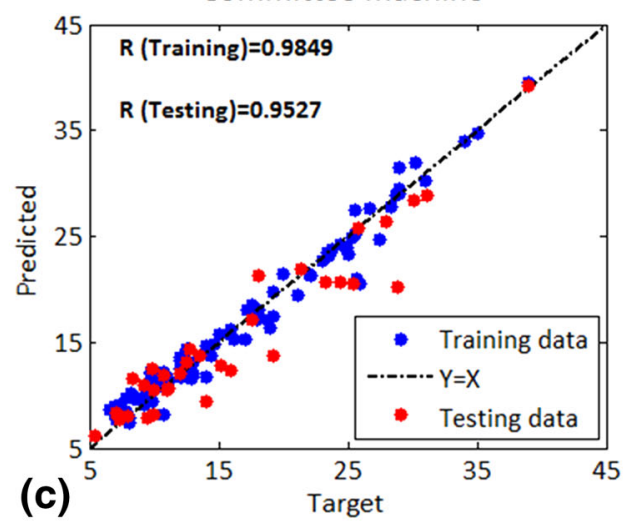

Fig. 5 Comparison between measured and ONN predicted residual shear strength of clay in testing data

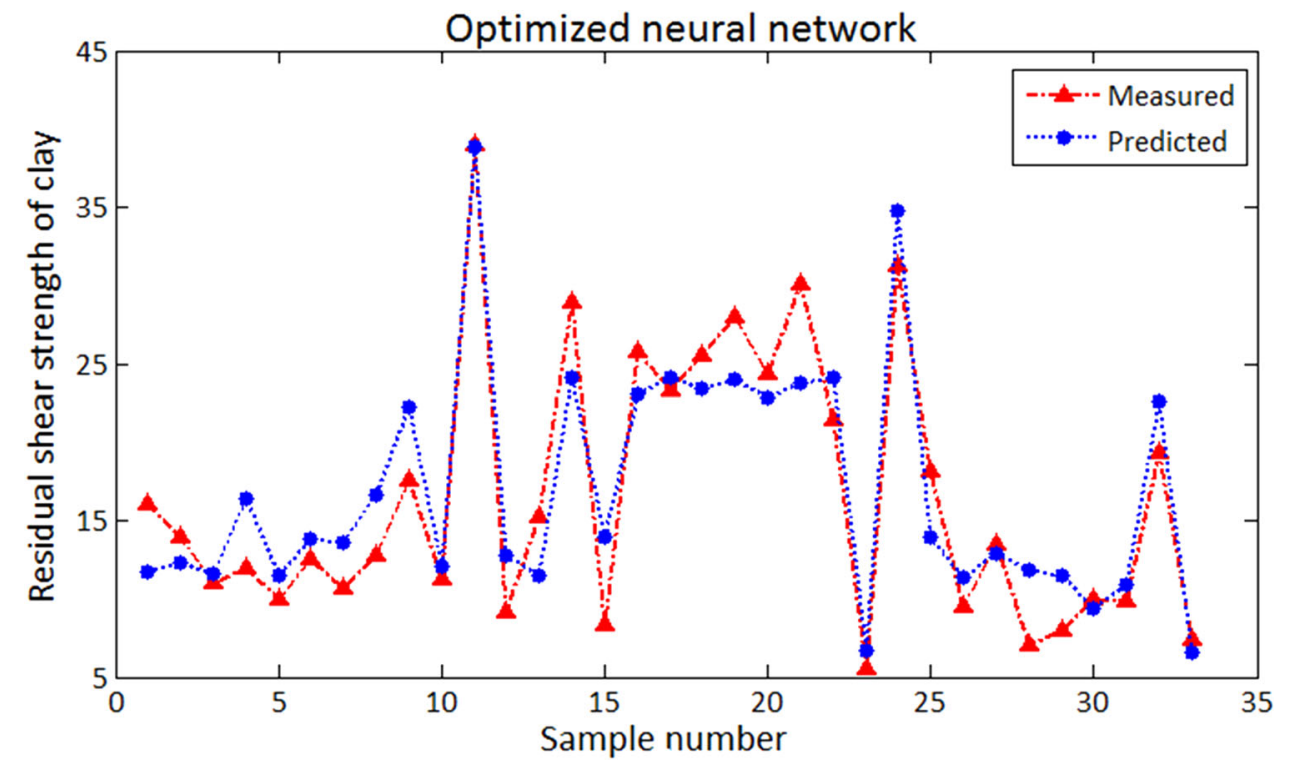

\section{Optimized fuzzy logic}

Regarding to previous studies, accurate determination of fuzzy parameters has a great influence on forecasting performance of fuzzy logic. Hence, these parameters must be optimized to gain model with excellent predictive performance. In this study, fuzzy logic is optimized with BA and this optimized model is adopted to correlating of RSS of clay to its index properties. In Table 4 , regulations which must be done before running of BA for optimizing of FL 
Table 4 Optimal weighs of CM elements which achieved through BA algorithm

\begin{tabular}{ll}
\hline PLCM & \\
\hline Parameter & Value \\
\hline$\alpha_{1}$ & 0.884090879475210 \\
$\alpha_{2}$ & 0.163911094533168 \\
$\alpha_{3}$ & 1.214659043334770 \\
$\beta_{1}$ & 0.579188328114643 \\
$\beta_{2}$ & 1.424991194176970 \\
\hline
\end{tabular}

model are given. Figure $3 \mathrm{~b}$ show the running of BA for optimizing of FL model. The effect of clustering radius on accuracy of fuzzy based model is shown in Fig. 6, which indicates that by election value of 0.4 for clustering radius, the model with supreme performance is achieved. Figure 7 illustrated the membership function of input parameters which extracted after running BA. Figure $4 \mathrm{~b}$ demonstrated the extent of match between measured and estimated RSS of clay is term of scatter plot. This figure prove the acceptable correlation between measured and estimated RSS of clay. Figure 8 depicted different between actual and estimated RSS of clay versus sample number for testing dataset. It is revealed that the predicted results agree well with the real value of RSS of clay. Results of this section concluded that OFL can be successfully applied to establish accurate and reliable prediction models for estimation of RSS of clay.

\section{Committee machine}

In this study, two optimized models are constructed thus far, and those outputs are determined. Both methods seemed to be useful, although a comparison between the two methods revealed the slight superiority of the OFL over the ONN model. As described above, in this stage, outputs of optimized models are gathered and integrated through $\mathrm{CM}$ for produced models which reaping those benefits. BA is included in $\mathrm{CM}$ for computation of optimal

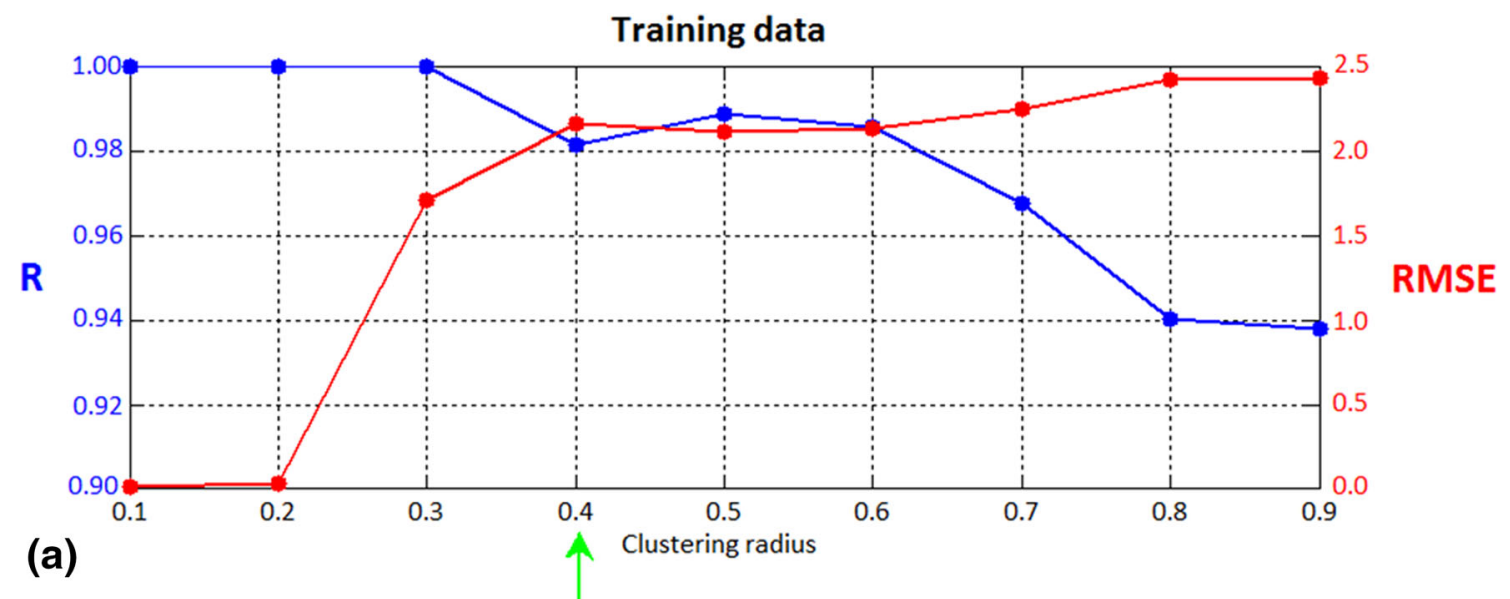

(a)

Testing data

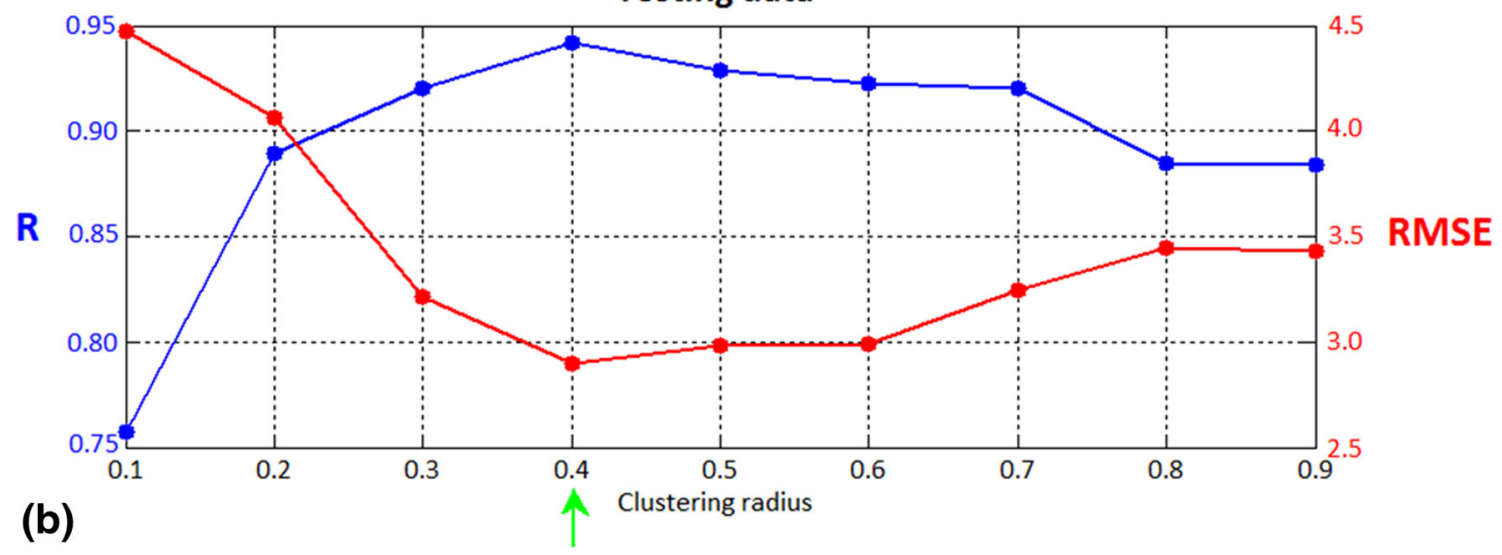

Fig. 6 The variation of accuracy of OFL models with respect to the clustering radius for a training data and $\mathbf{b}$ testing data. As seen in this figure, 0.4 is suitable value for clustering radius in order to constructing optimized fuzzy logic 

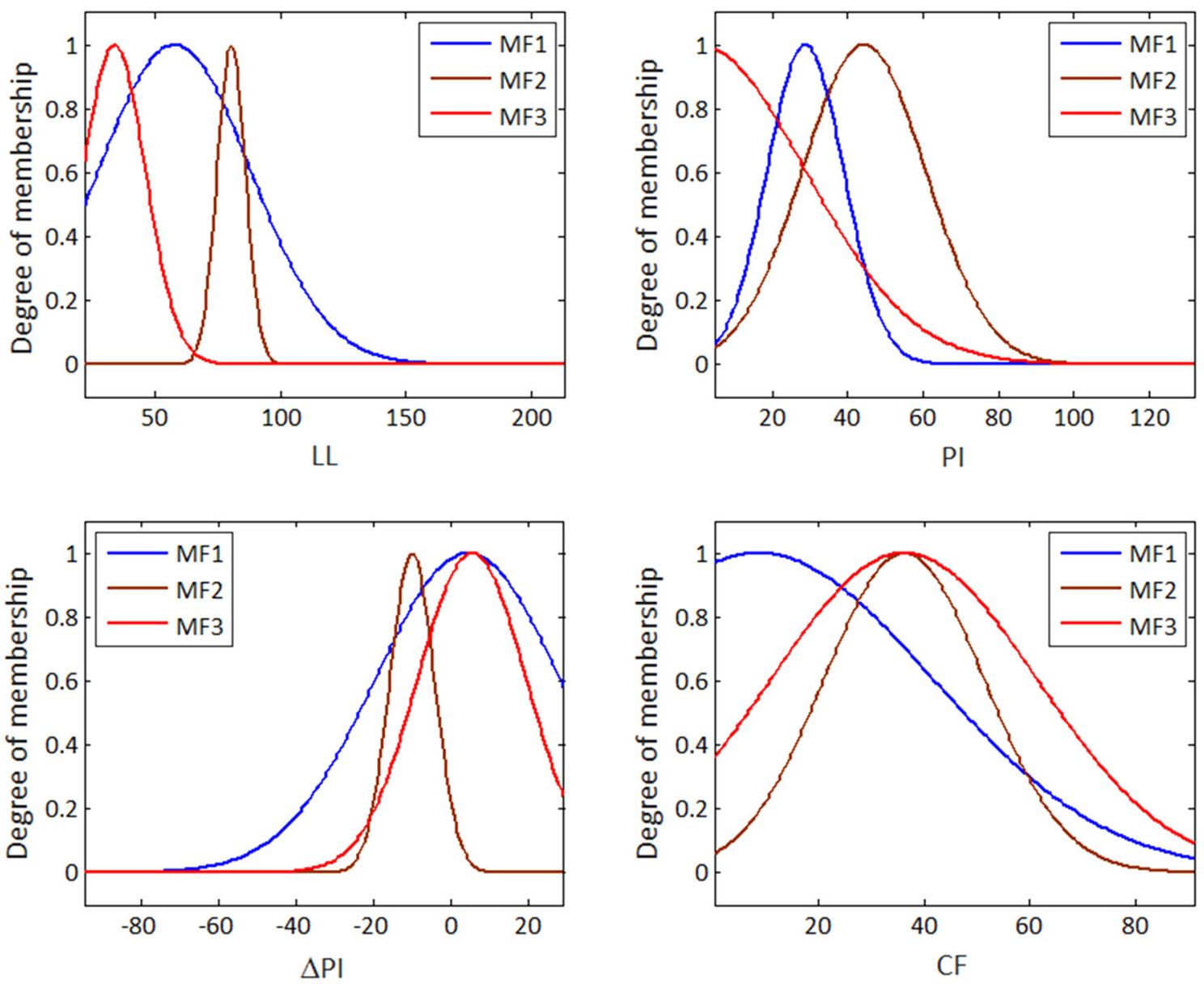

Fig. 7 Membership function for input parameters of optimized fuzzy logic model

Fig. 8 A typical comparison between predicted residual shear strength of clay by OFL and the corresponding experimental data for testing dataset

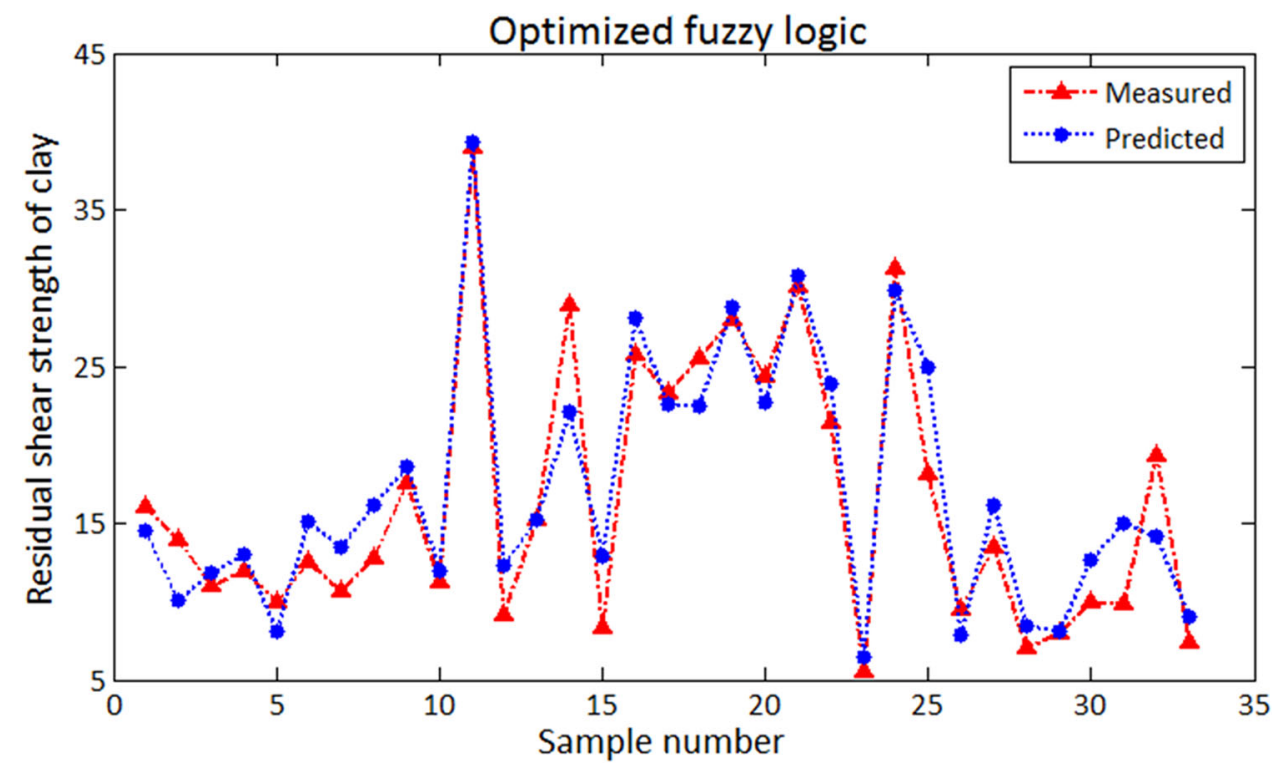

weights of optimized models. These assigned weights reflect optimal contribution of optimized models in final prediction according to their corresponding performance.
For achieving aforementioned objective (determining the optimal contribution of optimized models in final prediction), following fitness function is introduced to BA. 
Fig. 9 Comparison between measured and $\mathrm{CM}$ predicted residual shear strength of clay in testing data

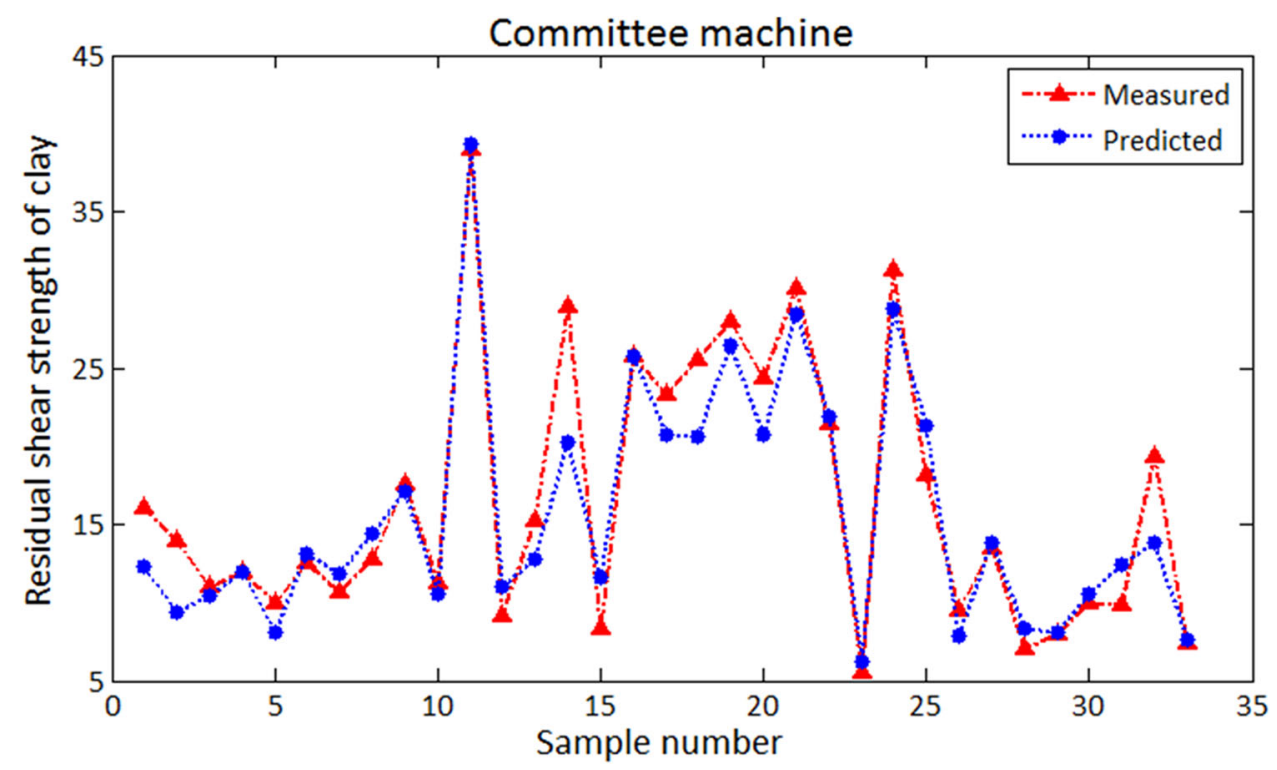

Table 5 Comparison between the obtained results using the CM, ONN and OFL models based on statistical index

\begin{tabular}{llll}
\hline Model & Allocation & $R$ & RMSE \\
\hline ONN & Training & 0.9816 & 2.7038 \\
& Testing & 0.9284 & 3.1646 \\
& Total & 0.9620 & 2.8261 \\
OFL & Training & 0.9818 & 2.1651 \\
& Testing & 0.9415 & 2.8981 \\
& Total & 0.9695 & 2.3697 \\
CM & Training & 0.9849 & 1.3757 \\
& Testing & 0.9527 & 2.7288 \\
& Total & 0.9745 & 1.8113 \\
\hline
\end{tabular}

$\mathrm{MSE}_{\mathrm{CM}}=\frac{1}{k} \sum_{i=1}^{k}\left(\alpha_{1} \times \mathrm{ONN}^{\beta_{1}}+\alpha_{2} \times \mathrm{OFL}^{\beta_{2}}+\alpha_{3}-T_{i}\right)^{2}$

where $\alpha_{i}$ and $\beta_{i}$ are constant coefficients which computed with BA. In Table 4, regulations which must be done before running of BA for computing of optimal weight of predictive models are given. Figure $3 \mathrm{c}$ is show the running of BA for determining the accurate values of optimized models weight factors. The BA-determined weights of optimized models is given in Table 2 . Figure $4 \mathrm{c}$ shows the predicted versus experimental values for the training and testing sets with CM method. This figure demonstrated that the values obtained from CM are very close to the experimental results. Moreover, a comparison of the scatter plot in Fig. 4 shows that the results of $\mathrm{CM}$ has better results in comparison to OFL and ONN. Comparison of actual and predicted RSS of clay versus sample number of testing data are demonstrated in Fig. 9. From this figure, it is clearly observed that committee model results have good agreement with real data. In this study, statistical criteria including $R$ and RMSE are used to assess the predicting performance of CM compared with that of OFL and ONN. Table 5 reports the result of statistical quality measures of $\mathrm{CM}$ and its elements (ONN and OFL) for prediction of RSS of clay. As can be obviously seen in Table 5, CM give the smallest error and highest correlation coefficient compared to optimized model in relating the RSS of clay to it index properties.

\section{Conclusions}

Estimation of RSS of clay is worthwhile because of it play a vital role in landslide stability. In this study, a smart model based on assembling of optimized elements (ONN and OFL) has been developed for constructing a quantitative relation between the RSS of clay and readily available clay index. The ONN and OFL models was obtained through embedding of the $\mathrm{BA}$ in those formulation. Moreover, BA is employed for computing the optimal contribution of optimized models in final prediction. The CM estimates were compared with those of the optimized models (ONN and OFL models) and the following conclusions can be drawn:

1. With the BA, the parameters of $\mathrm{NN}$ and FL are optimized and those predictive performance are enhanced.

2. ONN is potent model for determining the RSS of clay. 
3. OFL exhibit high level of performance and generalization ability in modeling of the RSS of clay. This model showed slightly better performance than the ONN model.

4. Better performance of $\mathrm{CM}$ compared to its members (ONN and OFL) are proved by lower RMSE and higher $R$ in results.

5. The $\mathrm{CM}$ can satisfactorily be used in obtaining the RSS of clay, therefore the cost and time for experimentally measurement of this parameter in laboratory would be saved.

\section{References}

Afshar M, Gholami A, Asoodeh M (2014) Genetic optimization of neural network and fuzzy logic for oil bubble point pressure modeling. Korean J Chem Eng 31(3):496-502

Ansari HR (2014) Use seismic colored inversion and power law committee machine based on imperial competitive algorithm for improving porosity prediction in a heterogeneous reservoir. J Appl Geophys 108:61-68

Ansari HR, Gholami A (2015) An improved support vector regression model for estimation of saturation pressure of crude oils. Fluid Phase Equilib 402:124-132

Asoodeh M, Bagheripour P (2012a) Estimation of bubble point pressure from PVT data using a power-law committee with intelligent systems. J Pet Sci Eng 90-91:1-11

Asoodeh M, Bagheripour P (2012b) Prediction of compressional, shear, and Stoneley wave velocities from conventional well log data using a committee machine with intelligent systems. Rock Mech Rock Eng 45(1):45-63

Asoodeh M, Gholami A, Bagheripour P (2014a) Oil- $\mathrm{CO}_{2} \mathrm{MMP}$ determination in competition of neural network, support vector regression, and committee machine. J Dispers Sci Technol 35(4):564-571

Asoodeh M, Gholami A, Bagheripour P (2014b) Asphaltene precipitation of titration data modeling through committee machine with stochastically optimized fuzzy logic and optimized neural network. Fluid Phase Equilib 364:67-74

Asoodeh M, Bagheripour P, Gholami A (2015) NMR parameters determination through ACE committee machine with genetic implanted fuzzy logic and genetic implanted neural network. Acta Geophys 63(3):735-760

Baughman DR, Liu YA (1985) Neural networks in bioprocessing and chemical engineering. Academic Press, London

Clague JJ, Stead D (2012) Landslides: types, mechanisms and modeling. Cambridge University Press, London

Colotta T, Cantoni R, Pavesi U, Robert E, Moretti PC (1989) A correlation between residual friction angle, gradation and index properties of cohesive soil. Geotechnique 39:343-346

Das SK, Basudhar PK (2008) Prediction of residual friction angle of clays using artificial neural network. Eng Geol 100(3-4):142-145
Das SK, Samui P, Khan SZ, Sivakugan N (2011) Machine learning techniques applied to prediction of residual strength of clay. Cent Eur J Geosci 3(4):449-461

Gholami A, Asoodeh M, Bagheripour P (2014a) How committee machine with SVR and ACE estimates bubble point pressure of crudes. Fluid Phase Equilib 382:139-149

Gholami A, Asoodeh M, Bagheripour P (2014b) Smart determination of difference index for asphaltene stability evaluation. J Dispers Sci Technol 35(4):572-576

Gholami A, Asoodeh M, Bagheripour P (2014c) Fuzzy assessment of asphaltene stability in crude oils. J Dispers Sci Technol 35(4):556-563

Gholami A, Afshar M, Bagheripour P, Asoodeh M, Vaezzadeh-Asadi M (2015a) Smart correlation of compositional data to saturation pressure. J Nat Gas Sci Eng 22:661-669

Gholami A, Mohammadzadeh O, Kord S, Moradi S, Dabir B (2015b) Improving the estimation accuracy of titration-based asphaltene precipitation through power-law committee machine (PLCM) model with alternating conditional expectation (ACE) and support vector regression. J Pet Explor Prod Technol. doi:10. 1007/s13202-015-0189-3

Kadkhodaie-Ilkhchi A, Rahimpour-Bonab H, Rezaee M (2009a) A committee machine with intelligent systems for estimation of total organic carbon content from petrophysical data: an example from Kangan and Dalan reservoirs in South Pars Gas Field, Iran. Comput Geosci 35:459-474

Kadkhodaie-Ilkhchi A, Rezaee M, Rahimpour-Bonab H (2009b) A committee neural network for prediction of normalized oil content from well log data: an example from South Pars Gas Field, Persian Gulf. J Pet Sci Eng 65(1-2):23-32

Kaya A, Kwong JKP (2007) Evaluation of common practice empirical procedures for residual friction angle of soils: Hawaiian amorphous material rich colluvial soil case study. Eng Geol 92(1-2):49-58

Khan SZ, Suman A, Pavani M, Das SK (2015) Prediction of the residual strength of clay using functional networks. Geosci Front. doi:10.1016/j.gsf.2014.12.008

Mäkisara K, Simula O, Kangas J, Kohonen T (2005) Artificial neural networks. Elsevier, Amsterdam

Mesri G, Shahien M (2003) Residual shear strength mobilized in firsttime slope failures. J Geotech Geoenviron Eng 129:12-31

Skempton AW (1964) The long term stability of clay slopes. Geotechnique 14:77-101

Tiwari B, Marui H (2005) A new method for the correlation of residual shear strength of the soil with mineralogical composition. J Geotech Geoenviron Eng 131:1139-1150

Wesley LD (2003) Residual strength of clays and correlations using Atterberg limit. Geotechnique 53:669-672

Yang XS (2010) A new metaheuristic bat-inspired algorithm. Stud Comput Intell 284:65-74

Zadeh LA (1965) Fuzzy sets. Inf Control 8(3):338-353

Zargar G, Bagheripour P, Asoodeh M, Gholami A (2015a) Oil- $\mathrm{CO}_{2}$ minimum miscible pressure (MMP) determination using a stimulated smart approach. Can J Chem Eng 93(10):1730-1735

Zargar G, Gholami A, Asoodeh M, Bagheripour P, Vaezzadeh-Asadi M (2015b) PSO-fuzzy eliminates deficiency of neuro-fuzzy in assessment of asphaltene stability. Indian $\mathrm{J}$ Chem Technol 22:135-140 